\title{
Relations among similarity measure, subsethood measure and fuzzy entropy
}

\author{
Yingfang Li, Keyun Qin, Xingxing He* \\ School of Mathematics, Southwest Jiaotong University, \\ Chengdu 610031, Sichuan, PR China \\ E-mail: yingfangli010@gmail.com,keyunqin@263.net,x.he@home.swjtu.edu.cn \\ Received 30 May 2012 \\ Accepted 12 January 2013
}

\begin{abstract}
In this paper we study the relations among similarity measure, subsethood measure and fuzzy entropy and present several propositions that similarity measure, subsethood measure and fuzzy entropy can be transformed by each other based on their axiomatic definitions. Some new formulae to calculate similarity measure, subsethood measure and fuzzy entropy are proposed.
\end{abstract}

Keywords: Similarity measure; Subsethood measure; Fuzzy entropy; Fuzzy equivalence

\section{Introduction}

In fuzzy set theory, similarity measure, subsethood measure and fuzzy entropy are three basic concepts. They surface in many fields, such as image processing, fuzzy neural networks, fuzzy reasoning, and fuzzy control.

The similarity measure describes the degree of similarity of fuzzy sets $A$ and $B$. Wang ${ }^{26}$ first put forward the concept of similarity measure of fuzzy sets and gave a computation formula. Since that time, many researchers began to contribute to the comparative study of similarity measures. For example, in 1993, Pappis et al. ${ }^{23}$ presented and compared the properties of several measures of similarity of fuzzy values. The work of Pappis ${ }^{23}$ was extended by Chen et al. ${ }^{8}$ and Wang et al. ${ }^{27}$. Fan et al. ${ }^{11}$ gave a general definition of similarity measure and discussed some properties of similarity measure. Bustince et al. ${ }^{5}$ proposed the concept of restricted equivalence function and then used this function to construct similarity measure ${ }^{5,6}$.
The subsethood measure (also called inclusion measure) is a relation between fuzzy sets $A$ and $B$, which indicates the degree to which $A$ is contained in $B$. Traditionally, fuzzy set inclusion is defined according to Zadeh's ${ }^{31}$ original proposal. For $A$ and $B$ fuzzy sets in a universe $X$ he defined: $A \subseteq B$ iff for all $x \in X, A(x) \leqslant B(x)$. For many researchers, this definition is too rigid and it does not do justice to the spirit of the Theory of Fuzzy Sets $1,10,20$. Because of that a great number of fuzzy alternatives to Zadeh's original operator have been suggested in the literature. So far, four axiomatizations have been given for subsethood measures. The first one was given by $\mathrm{Ki}$ tainik ${ }^{16}$ in 1987 . Then Sinha and Dougherty ${ }^{24}$ presented nine axioms for subsethood measures, plus three additional ones. Young ${ }^{30}$ gave four axioms for these measures. Later, Fan et al. ${ }^{12}$ modified one of Young's axioms. Finally, in 2006 Bustince et al. ${ }^{4}$ modified two of Young's axioms and proposed a new class of subsethood measure called fuzzy $D I$ subsethood measure.

\footnotetext{
${ }^{*}$ Corresponding author.
} 
The entropy of a fuzzy set is the fuzziness of that set. A measure of entropy indicates the degree to which a set is fuzzy. It is therefore a fuzzy set in $F(X)$. Several researchers have studied fuzzy entropy measure from different points of view. In 1972, De Luca and Termini ${ }^{9}$ introduced some axioms that capture our intuitive comprehension to describe the fuzziness degree of a fuzzy set. Kaufmann 15 presented a method to measure the fuzziness degree of a fuzzy set by a metric distance between its membership function and the membership function of its nearest crisp set. Yager ${ }^{29}$ viewed the fuzziness degree of a fuzzy set in terms of a lack of distinction between the fuzzy set and its complement. Trillas and Riera ${ }^{25}$ proposed general expressions for the entropy given by Yager. Loo ${ }^{22}$ gave a definition which contained those given by De Luca and Kaufmann. Burillo and Bustince ${ }^{2}$ studied the concepts of entropy for intuitionistic fuzzy sets and intervalvalued fuzzy sets.

In addition to their concepts, many researchers have contributed to discussing the relations among the above-mentioned three concepts. For example, in 1986 Kosko ${ }^{18}$ showed that the entropy of a fuzzy set $A$ is the degree to which $A \cup A^{c}$ is a subset of its complement $\left(A \cup A^{c}\right)^{c}=A \cap A^{c}$. He also showed 19 that the classical probability of a crisp event $C$ is the degree to which the universal set $X$ is a subset of $C$. Then Young ${ }^{30}$ linked fuzzy entropy, subsethood and probability in a more general setting. Liu ${ }^{21}$ discussed the relations among fuzzy entropy, distance measure, and similar- ity measure of fuzzy sets. Fan ${ }^{11}$ gave a simplified expression of similarity measure induced by fuzzy entropy. Zeng et al. ${ }^{32}$ investigated the relations among subsethood measure, similarity measure, and fuzzy entropy based on their axiomatic definitions.

Notice that, the present paper is related to, but different from the above-mentioned works, it focuses on discussing the relationships among subsethood measure, similarity measure, and fuzzy entropy in a more general setting and can be thought of as an extension of the above works. We propose several propositions to show that these three concepts can be transformed by each other based on their axiomatic definitions. Some new formulae to calculate similarity measure, subsethood measure and fuzzy entropy are also given. We can later see that the results obtained in the above-mentioned works can be brought into line with the present work.

Throughout this paper, we write $X$ to denote the universal set, $F(X)$ stands for the set of fuzzy sets in $X, P(X)$ stands for the set of crisp sets in $X$. We assume that $X$ is a finite set here. One can readily obtain our results for $X$ infinite. We use capital letters $A, B, C$ to denote fuzzy sets on $X$ and write $A(x), B(x), C(x)$ for their membership functions, respectively. Define $A^{c}(x)=1-A(x)$ for all $x \in X$; we call $A^{c}$ the complement of $A$. Let $\left[\frac{1}{2}\right]$ stand for the fuzzy set of $X$ for which $\left[\frac{1}{2}\right](x)=\frac{1}{2}$ for all $x \in X$.

\section{Relation between similarity measure and subsethood measure}

Firstly we present several concepts of fuzzy set theory that are necessary for our considerations.

Definition 1. ${ }^{17}$ A function $n:[0,1] \longrightarrow[0,1]$ is called a fuzzy negation if it satisfies:

(n1) $n(0)=1$ and $n(1)=0$.

(n2) If $x \leqslant y$, then $n(x) \geqslant n(y)$.

A fuzzy negation is said to be involutive if

(n3) $n(n(x))=x$ for all $x \in[0,1]$.

Definition 2. ${ }^{3}$ A continuous, strictly increasing function $\varphi:[a, b] \longrightarrow[a, b]$ with boundary conditions $\varphi(a)=a, \varphi(b)=b$ is called an automorphism of the interval $[a, b]$.

Definition 3. ${ }^{17}$ An associative, commutative and increasing function $T:[0,1]^{2} \longrightarrow[0,1]$ is called a $t-$ norm if it has the neutral element equal to 1 .

An associative, commutative and increasing function $S:[0,1]^{2} \longrightarrow[0,1]$ is called a t-conorm if it has the neutral element equal to 0 .

Example 1. ${ }^{17}$ Table 1 lists several t-norms and tconorms used extensively in this paper.

Fodor and Roubens define fuzzy equivalence as a binary operation on the unit interval in the following way ${ }^{14}$. 
Table 1: Several t-norms and t-conorms

\begin{tabular}{llll}
\hline T-norms & Formulae of t-norms & T-conorms & Formulae of t-conorms \\
\hline$T_{M}$ & $\min (x, y)$ & $S_{M}$ & $\max (x, y)$ \\
$T_{P}$ & $x y$ & $S_{P}$ & $x+y-x y$ \\
$T_{L}$ & $\max (x+y-1,0)$ & $S_{L}$ & $\min (x+y, 1)$ \\
\hline
\end{tabular}

Definition 4. ${ }^{14}$ A function $E:[0,1]^{2} \longrightarrow[0,1]$ is called a fuzzy equivalence if it satisfies the following properties:

(E1) $E(x, y)=E(y, x)$ for all $x, y \in[0,1]$.

(E2) $E(x, x)=1$ for all $x \in[0,1]$.

(E3) $E(0,1)=E(1,0)=0$.

(E4) For all $x, y, x^{\prime}, y^{\prime} \in[0,1]$, if $x \leqslant x^{\prime} \leqslant y^{\prime} \leqslant y$, then $E(x, y) \leqslant E\left(x^{\prime}, y^{\prime}\right)$.

We can prove that E4 is equivalent to: for all $x, y, z \in[0,1]$, if $x \leqslant y \leqslant z$, then $\min (E(x, y), E(y, z)) \geqslant$ $E(x, z)$. The following reasonable properties can be considered for the fuzzy equivalence: for all $x, y \in$ $[0,1]$,

(E5) $E(x, y)=1$ iff $x=y$.

(E6) $E(x, 1-x)=0$ iff $x=0$ or $x=1$.

In 2006, Bustince et al. ${ }^{5}$ proposed the concept of restricted equivalence function, which arises from the concept of fuzzy equivalence and from the properties usually demanded from the measures used for comparing images 6,7 .

Definition 5. ${ }^{5}$ A function $R E F:[0,1]^{2} \longrightarrow[0,1]$ is called a restricted equivalence function, if it satisfies the following properties:

(1) $\operatorname{REF}(x, y)=\operatorname{REF}(y, x)$ for all $x, y \in[0,1]$.

(2) $\operatorname{REF}(x, y)=1$ iff $x=y$.

(3) $\operatorname{REF}(x, y)=0$ iff $x=1$ and $y=0$ or $x=0$ and $y=1$.

(4) $\operatorname{REF}(x, y)=\operatorname{REF}(n(x), n(y))$ for all $x, y \in[0,1]$, $n$ being a strong fuzzy negation.
(5) For all $x, y, z \in[0,1]$, if $x \leqslant y \leqslant z$, then $R E F(x, y) \geqslant \operatorname{REF}(x, z)$ and $\operatorname{REF}(y, z) \geqslant$ $\operatorname{REF}(x, z)$.

Proposition 1. ${ }^{5}$ If $\varphi$ is an automorphism of the unit interval, then

$R E F(x, y)=\varphi^{-1}(1-|\varphi(x)-\varphi(y)|)$ with $n(x)=\varphi^{-1}(1-\varphi(x))$

is a restricted equivalence function.

It is easy to obtain the following conclusion from Proposition 1.

Proposition 2. If $\varphi$ is an automorphism of the unit interval, then $E(x, y)=\varphi^{-1}(1-|\varphi(x)-\varphi(y)|)$ is a fuzzy equivalence satisfying $E 5$ and $E 6$.

Proposition 3. If $\varphi$ is an automorphism of the unit interval, then $E(x, y)=\varphi^{-1}\left(\frac{\min (\varphi(x), \varphi(y))}{\max (\varphi(x), \varphi(y))}\right)$ is a fuzzy equivalence satisfying $E 5$ and $E 6$.

Proof. It is easy to prove that E satisfies E1E3 and E5-E6. We only prove that E4 holds. If $x \leqslant y \leqslant z$, then $\varphi(x) \leqslant \varphi(y) \leqslant \varphi(z)$. Therefore, we have $\frac{\varphi(x)}{\varphi(y)} \geqslant \frac{\varphi(x)}{\varphi(z)}$ and $\frac{\varphi(y)}{\varphi(z)} \geqslant \frac{\varphi(x)}{\varphi(z)}$. Thus, $E(x, y)=\varphi^{-1}\left(\frac{\varphi(x)}{\varphi(y)}\right) \geqslant \varphi^{-1}\left(\frac{\varphi(x)}{\varphi(z)}\right)=E(x, z)$ and $E(y, z)=$ $\varphi^{-1}\left(\frac{\varphi(y)}{\varphi(z)}\right) \geqslant \varphi^{-1}\left(\frac{\varphi(x)}{\varphi(z)}\right)=E(x, z)$.

Next we discuss the relation between similarity measure and subsethood measure and propose several propositions that similarity measure and subsethood measure can be transformed by each other based on their axiomatic definitions.

In 1983, Wang ${ }^{26}$ introduced the concept of similarity measure of fuzzy sets and gave a formula to calculate the lattice similarity measure of fuzzy sets. Some formulae to calculate similarity measures were also introduced in ${ }^{8,11,23,27}$. Actually, if the definition of fuzzy equivalence is extended to sets, we can obtain the definition of similarity measure. 
Definition 6. ${ }^{26}$ A function $N: F(X) \times F(X) \longrightarrow$ $[0,1]$ is called a similarity measure if it satisfies the following properties:

(N1) $N(X, \emptyset)=0$ and $N(A, A)=1$ whenever $A \in$ $F(X)$.

(N2) $N(A, B)=N(B, A)$ whenever $A, B \in F(X)$.

(N3) For all $A, B, C \in F(X), \quad N(A, C) \leqslant$ $\min (N(A, B), N(B, C))$ whenever $A \subseteq B \subseteq C$.

In the following, we present some further axioms in terms of similarity measure $N$. Some of these properties are required in different papers and can also be important in some applications.

(N4) $N(A, B)=1$ iff $A=B$ for all $A, B \in F(X)$.

(N5) $N(A, B)=0$ iff $A \cap B=\emptyset$ and $A \cup B \neq \emptyset$ for all $A, B \in F(X)$.

(N6) If $A \subseteq B$, then $N(A \cup C, A) \leqslant N(B \cup C, B)$ for all $A, B, C \in F(X)$.

(N7) If $A \subseteq B$, then $N(A, A \cap C) \geqslant N(B, B \cap C)$ for all $A, B, C \in F(X)$.

(N8) $N\left(A, A \cap A^{c}\right)=0$ iff $A=X$ for all $A \in F(X)$.

(N9) $N\left(A \cup A^{c}, A^{c}\right)=0$ iff $A=X$ for all $A \in F(X)$.

In the previous part of this paper, we have referred to four axiomatizations of subsethood measures. We will discuss the last three ones here.

On the basis of Kosko's ${ }^{18,19}$ subsethood measure, fuzzy entropy and Wilmott's work ${ }^{28}$, Young defined subsethood measure in the following way:

Definition 7. ${ }^{30}$ A function $c_{V Y}: F(X) \times F(X) \longrightarrow$ $[0,1]$ is called a $V Y$-subsethood measure, if $c_{V Y}$ satisfies the following conditions:

(C1) $c_{V Y}(A, B)=1$ iff $A \subseteq B$, i.e., $A(x) \leqslant B(x)$ for all $x \in X$.

(C2) If $\left[\frac{1}{2}\right] \subseteq A$, then $c_{V Y}\left(A, A^{c}\right)=0$ iff $A=X$.

(C3) If $A \subseteq B \subseteq C$, then $c_{V Y}(C, A) \leqslant c_{V Y}(B, A)$ and if $A \subseteq B$, then $c_{V Y}(C, A) \leqslant c_{V Y}(C, B)$.
It has been pointed out in ${ }^{12}$ that $\mathrm{C} 3$ is too strong when considering the relation between subsethood measure and fuzzy entropy. Therefore, Fan et al. ${ }^{12}$ thought a simpler form of definition of subsethood measure based on Young's definition.

Definition 8. ${ }^{12}$ A function $c_{*}: F(X) \times F(X) \longrightarrow$ $[0,1]$ is called a $*$-subsethood measure, if $c_{*}$ satisfies the following conditions:

(C1) $c_{*}(A, B)=1$ iff $A \subseteq B$, i.e., $A(x) \leqslant B(x)$ for all $x \in X$.

(C2) If $\left[\frac{1}{2}\right] \subseteq A$, then $c_{*}\left(A, A^{c}\right)=0$ iff $A=X$.

(C3) If $A \subseteq B \subseteq C$, then $c_{*}(C, A) \leqslant c_{*}(B, A)$ and $c_{*}(C, A) \leqslant c_{*}(C, B)$.

Obviously, the only difference between Definitions 7 and 8 is in $\mathrm{C} 3$ where Young demands increasingness in the second component. Thus every $V Y$-subsethood measure is also a $*$-subsethood measure. In 2006, Bustince et al. ${ }^{4}$ modified two of Young's axioms and proposed a new class of subsethood measure called $D I$-subsethood measure.

Definition 9. ${ }^{4}$ A function $c_{D I}: F(X) \times F(X) \longrightarrow$ $[0,1]$ is called a $D I$-subsethood measure, if $c_{D I}$ satisfies the following conditions:

(C1) $c_{D I}(A, B)=1$ iff $A \subseteq B$, i.e., $A(x) \leqslant B(x)$ for all $x \in X$.

(C2) $c_{D I}\left(A, A^{c}\right)=0$ iff $A=X$.

(C3) If $A \subseteq B$, then $c_{D I}(A, C) \geqslant c_{D I}(B, C)$ and $c_{D I}(C, A) \leqslant c_{D I}(C, B)$.

It is shown that every $D I$-subsethood measure is a $V Y$-subsethood measure and therefore, it is also a *-subsethood measure.

Considering that an axiom definition must generally be abstract and simple, Fan ${ }^{12}$ gave the following definition.

Definition 10. ${ }^{12}$ A function $c: F(X) \times F(X) \longrightarrow$ $[0,1]$ is called a subsethood measure, if $c$ satisfies the following conditions:

(C1) If $A \subseteq B$, then $c(A, B)=1$.

(C2) $c(X, \emptyset)=0$.

(C3) If $A \subseteq B \subseteq C$, then $c(C, A) \leqslant c(B, A)$ and $c(C, A) \leqslant c(C, B)$. 
We can see that $V Y$-subsethood measure, *subsethood measure and $D I$-subsethood measure are special cases of subsethood measures. In the following we derive subsethood measures from similarity measures.

Proposition 4. Given a discrete universe $X=$ $\left\{x_{1}, x_{2}, \ldots, x_{n}\right\}$. Let $N$ be a similarity measure, $c$ a function defined for all $A, B \in F(X)$ by $c(A, B)=$ $N(A, A \cap B)$. Then we have the following conclusions:

(1) c is a subsethood measure.

(2) If $N$ satisfies $N 4$ and $N 8$, then $c$ is a VYsubsethood measure.

(3) If $N$ satisfies $N 4$ and $N 8$, then $c$ is $a *-$ subsethood measure.

(4) If $N$ satisfies $N 4$ and $N 5$, then $c$ is a VYsubsethood measure.

(5) If $N$ satisfies $N 4$ and N5, then $c$ is $a *-$ subsethood measure.

(6) If $N$ satisfies N4, N7 and N8, then $c$ is a DIsubsethood measure.

\section{Proof.}

(1) (C1) If $A \subseteq B$, then $c(A, B)=N(A, A \cap B)=$ $N(A, A)=1$.

(C2) $c(X, \emptyset)=N(X, X \cap \emptyset)=N(X, \emptyset)=0$.

(C3) If $A \subseteq B \subseteq C$, then $c(C, A)=N(C, C \cap$ $A)=N(C, A), c(B, A)=N(B, B \cap A)=N(B, A)$, and $c(C, B)=N(C, C \cap B)=N(C, B)$. Since $N(C, A) \leqslant N(B, A)$, we have $c(C, A) \leqslant c(B, A)$. Since $N(C, A) \leqslant N(C, B)$, we have $c(C, A) \leqslant$ $c(C, B)$.

(2) (C1) The sufficiency has been proved in (1), we only prove the necessity here. If $c(A, B)=1$, then $N(A, A \cap B)=1$. By N4 we have $A=A \cap B$. Thus $A \subseteq B$ holds.

(C2) If $c\left(A, A^{c}\right)=0$, then $N\left(A, A \cap A^{c}\right)=0$. As $N$ satisfies $N 8$ and $\left[\frac{1}{2}\right] \subseteq A$ we have $A=X$. On the contrary, if $A=X$, then $c\left(A, A^{c}\right)=N\left(A, A \cap A^{c}\right)=$ $N(X, \emptyset)=0$.
(C3) We only prove that $A \subseteq B$ implies $c(C, A) \leqslant$ $c(C, B)$ here. If $A \subseteq B$, then $C \cap A \subseteq C \cap B \subseteq$ $C$, we have $c(C, A)=N(C, C \cap A), c(C, B)=$ $N(C, C \cap B)$. Since $N(C, C \cap A) \leqslant N(C, C \cap B)$, then $c(C, A) \leqslant c(C, B)$.

(3) It follows directly from (2).

(4) We only prove that the necessity of $C 2$ also holds when $N$ satisfies $N 5$. If $\left[\frac{1}{2}\right] \subseteq A$, then $c\left(A, A^{c}\right)=$ $N\left(A, A \cap A^{c}\right)=N\left(A, A^{c}\right)=0$. As $N$ satisfies $N 5$ we have $A \cap A^{c}=\emptyset$, that is $A^{c}=\emptyset$, thus $A=X$.

(5) It follows directly from (4).

(6) It can be proved in the same manner with (2).

Example 2. Consider the following two similarity measures:

$$
\begin{aligned}
& N_{1}(A, B)=\frac{1}{n} \sum_{i=1}^{n} \frac{\min \left(A\left(x_{i}\right), B\left(x_{i}\right)\right)}{\max \left(A\left(x_{i}\right), B\left(x_{i}\right)\right)} . \\
& N_{2}(A, B)=\frac{\sum_{i=1}^{n} \min \left(A\left(x_{i}\right), B\left(x_{i}\right)\right)}{\sum_{i=1}^{n} \max \left(A\left(x_{i}\right), B\left(x_{i}\right)\right)} .
\end{aligned}
$$

It is shown that $N_{1}$ satisfies properties $\mathrm{N} 4, \mathrm{~N} 6$, $\mathrm{N} 7, \mathrm{~N} 8, \mathrm{~N} 9$ and $N_{2}$ satisfies properties N4, N5, N6. By Proposition 4, we obtain the following subsethood measures:

$$
\begin{aligned}
& c_{1}(A, B)=\frac{1}{n} \sum_{i=1}^{n} \frac{\min \left(A\left(x_{i}\right), B\left(x_{i}\right)\right)}{A\left(x_{i}\right)} . \\
& c_{2}(A, B)=\frac{\sum_{i=1}^{n} \min \left(A\left(x_{i}\right), B\left(x_{i}\right)\right)}{\sum_{i=1}^{n} A\left(x_{i}\right)} .
\end{aligned}
$$

We can conclude that $c_{1}$ is a $D I$-subsethood measure and therefore, it is also a $V Y$-subsethood measure and $*$-subsethood measure. We also conclude that $c_{2}$ is a $V Y$-subsethood measure and *subsethood measure.

Proposition 5. Given a discrete universe $X=$ $\left\{x_{1}, x_{2}, \ldots, x_{n}\right\}$. Let $N$ be a similarity measure, $c$ a function defined for all $A, B \in F(X)$ by $c(A, B)=$ $N(A \cup B, B)$. Then we have the following conclusions:

(1) c is a subsethood measure. 
(2) If $N$ satisfies $N 4, N 6$ and $N 9$, then $c$ is a VYsubsethood measure.

(3) If $N$ satisfies $N 4$ and N9, then $c$ is $a *-$ subsethood measure.

(4) If $N$ satisfies $N 4, N 5$ and N6, then $c$ is a $V Y$ subsethood measure.

(5) If $N$ satisfies $N 4$ and N5, then $c$ is $a *$ subsethood measure.

(6) If $N$ satisfies N4, N6 and N9, then $c$ is a DIsubsethood measure.

Proof. It can be proved in the same manner with Proposition 4.

Example 3. Consider the following two similarity measures:

$$
\begin{aligned}
& N_{3}(A, B)=\frac{\sum_{i=1}^{n}\left(1-\left|A\left(x_{i}\right)-B\left(x_{i}\right)\right|\right)}{\sum_{i=1}^{n}\left(1+\left|A\left(x_{i}\right)-B\left(x_{i}\right)\right|\right)} . \\
& N_{4}(A, B)=\frac{\sum_{i=1}^{n} 2 \min \left(A\left(x_{i}\right), B\left(x_{i}\right)\right)}{\sum_{i=1}^{n}\left(A\left(x_{i}\right)+B\left(x_{i}\right)\right)} .
\end{aligned}
$$

It is shown that $N_{3}$ satisfies properties N4, N6, N7, N8, N9 and $N_{4}$ satisfies properties N4, N5, N6. By Proposition 5, we obtain the following subsethood measures:

$$
\begin{aligned}
c_{3}(A, B) & =\frac{\sum_{i=1}^{n}\left(1-\left|\max \left(A\left(x_{i}\right), B\left(x_{i}\right)\right)-B\left(x_{i}\right)\right|\right)}{\sum_{i=1}^{n}\left(1+\left|\max \left(A\left(x_{i}\right), B\left(x_{i}\right)\right)-B\left(x_{i}\right)\right|\right)} . \\
c_{4}(A, B) & =\frac{\sum_{i=1}^{n} 2 B\left(x_{i}\right)}{\sum_{i=1}^{n}\left(\max \left(A\left(x_{i}\right), B\left(x_{i}\right)\right)+B\left(x_{i}\right)\right)} .
\end{aligned}
$$

We can conclude that $c_{3}$ is a $D I$-subsethood measure and therefore, it is also a $V Y$-subsethood measure and $*$-subsethood measure. We also conclude that $c_{4}$ is a $V Y$-subsethood measure and $*$ subsethood measure.

Proposition 6. Given a discrete universe $X=$ $\left\{x_{1}, x_{2}, \ldots, x_{n}\right\}$. Let $N$ be a similarity measure, $c$ a function defined for all $A, B \in F(X)$ by $c(A, B)=$ $N\left(A^{c}, A^{c} \cup B^{c}\right)$. Then we have the following conclusions:

(1) c is a subsethood measure.
(2) If $N$ satisfies $N 4$ and $N 9$, then $c$ is a $V Y$ subsethood measure.

(3) If $N$ satisfies $N 4$ and $N 9$, then $c$ is $a *-$ subsethood measure.

(4) If $N$ satisfies $N 4$ and $N 5$, then $c$ is a VYsubsethood measure.

(5) If $N$ satisfies $N 4$ and N5, then $c$ is $a *-$ subsethood measure.

(6) If $N$ satisfies N4, N6 and N9, then $c$ is a DIsubsethood measure.

Proof. It can be proved in the same manner with Proposition 4.

Proposition 7. Given a discrete universe $X=$ $\left\{x_{1}, x_{2}, \ldots, x_{n}\right\}$. Let $N$ be a similarity measure, $c$ a function defined for all $A, B \in F(X)$ by $c(A, B)=$ $N\left(B^{c}, A^{c} \cap B^{c}\right)$. Then we have the following conclusions:

(1) c is a subsethood measure.

(2) If $N$ satisfies N4, N7 and N8, then c is a VYsubsethood measure.

(3) If $N$ satisfies $N 4$ and N8, then $c$ is $a *-$ subsethood measure.

(4) If $N$ satisfies N4, N5 and N7, then $c$ is a VYsubsethood measure.

(5) If $N$ satisfies $N 4$ and $N 5$, then $c$ is $a *-$ subsethood measure.

(6) If $N$ satisfies N4, N7 and N8, then c is a DIsubsethood measure.

Proof. It can be proved in the same manner with Proposition 4.

In the following proposition we derive similarity measures from subsethood measures.

Proposition 8. Given a discrete universe $X=$ $\left\{x_{1}, x_{2}, \ldots, x_{n}\right\}$. Let $c$ be a subsethood measure, $T$ a t-norm, $N$ a function defined for all $A, B \in F(X)$ by $N(A, B)=T(c(A, B), c(B, A))$, then $N$ is a similarity measure.

Proof. 
(N1) $N(X, \emptyset)=T(c(X, \emptyset), c(\emptyset, X))=T(0,1)=0$. And $N(A, A)=T(c(A, A), c(A, A))=T(1,1)=1$.

(N2) $N(A, B)=T(c(A, B), c(B, A))=T(c(B, A), c(A, B))=$ $N(B, A)$.

(N3) If $A \subseteq B \subseteq C$, then $N(A, C)=$ $T(c(A, C), c(C, A))=c(C, A), \quad N(A, B)=$ $T(c(A, B), c(B, A))=c(B, A)$, and $N(B, C)=$ $T(c(B, C), c(C, B))=c(C, B)$. Since $c(C, A) \leqslant$ $c(B, A)$ and $c(C, A) \leqslant c(C, B)$, then $N(A, C) \leqslant$ $N(A, B)$ and $N(A, C) \leqslant N(B, C)$.

Corollary 9. In the conditions of Proposition 8, let $T=T_{P}$, then the similarity measure derived by subsethood measure can be expressed as: $N(A, B)=$ $c(A, B) \cdot c(B, A)$.

Corollary 10. In the conditions of Proposition 8, let $T=T_{M}$, then the similarity measure derived by subsethood measure can be expressed as: $N(A, B)=$ $\min (c(A, B), c(B, A))$.

Remark 1. We see that the two formulae given in Corollaries 9 and 10 are the same with the ones given by Zeng et al. in ${ }^{32}$. Hence Zeng's solutions can be seen as two special cases of ours.

Example 4. Consider the following subsethood measure:

$$
c_{5}(A, B)=\frac{1}{n} \sum_{i=1}^{n} \frac{\min \left(1-A\left(x_{i}\right), 1-B\left(x_{i}\right)\right)}{1-B\left(x_{i}\right)} .
$$

By Corollary 10, we obtain the following similarity measure:

$$
N_{5}(A, B)=\frac{\sum_{i=1}^{n} \min \left(1-A\left(x_{i}\right), 1-B\left(x_{i}\right)\right)}{\sum_{i=1}^{n} \max \left(1-A\left(x_{i}\right), 1-B\left(x_{i}\right)\right)} .
$$

\section{Relation between similarity measure and fuzzy entropy}

A measure of fuzzy entropy assesses the amount of vagueness, or fuzziness in a fuzzy set. De Luca and Termini ${ }^{9}$ formalize the properties of fuzzy entropy through the following axioms.
Definition 11. ${ }^{9}$ A function $e: F(X) \longrightarrow[0,1]$ is called an entropy on $F(X)$, if $e$ satisfies the following conditions:

(EP1) $e(A)=0$ iff $A$ is nonfuzzy, i.e., $A \in P(X)$.

(EP2) $e(A)=1$ iff $A=\left[\frac{1}{2}\right]$.

(EP3) $e(A) \leqslant e(B)$ if $A$ refines $B$, i.e., $A(x) \leqslant B(x)$ when $B(x) \leqslant \frac{1}{2}$ and $A(x) \geqslant B(x)$ when $B(x) \geqslant \frac{1}{2}$

(EP4) $e(A)=e\left(A^{c}\right)$.

Let $E$ be a fuzzy equivalence, $\mu$ a strictly decreasing function from $[0,1]$ to $\left[\frac{1}{2}, 1\right]$ with boundary conditions $\mu(0)=1, \mu(1)=\frac{1}{2}$. For fuzzy sets $A$ and $B$, we define $f(A, B) \in F(X)$, for all $x \in X$, $f(A, B)(x)=\mu(E(A(x), B(x)))$, then we have the following conclusion.

Proposition 11. Given a discrete universe $X=$ $\left\{x_{1}, x_{2}, \ldots, x_{n}\right\}$. Let e be a fuzzy entropy, $N$ a function defined for all $A, B \in F(X)$ by $N(A, B)=e(f(A, B))$, then $N$ is a similarity measure.

\section{Proof.}

(N1) For all $x \in X, f(X, \emptyset)(x)=\mu(E(X(x), \emptyset(x)))=$ $\mu(E(1,0))=\mu(0)=1$, then $f(X, \emptyset)=X$. Therefore, $N(X, \emptyset)=e(X)=0$. Note that $f(A, A)(x)=\mu(E(A(x), A(x)))=\mu(1)=\frac{1}{2}$, then $f(A, A)=\left[\frac{1}{2}\right]$. Thus $N(A, A)=e(f(A, A))=$ $e\left(\left[\frac{1}{2}\right]\right)=1$.

(N2) It is easy to see that $N(A, B)=e(f(A, B))=$ $e(f(B, A))=N(B, A)$.

(N3) $A \subseteq B \subseteq C$ implies $A(x) \leqslant B(x) \leqslant C(x)$ for all $x \in X$, by $E 4$, we have $E(A(x), C(x)) \leqslant$ $\min (E(A(x), B(x)), E(B(x), C(x))) . \quad B y$ the property of $\mu, \quad \mu(E(A(x), C(x))) \geqslant$ $\max (\mu(E(A(x), B(x))), \mu(E(B(x), C(x)))) \geqslant \frac{1}{2}$, i.e., $f(A, C)(x) \geqslant \max (f(A, B)(x), f(B, C)(x)) \geqslant$ $\frac{1}{2}$. By $\quad E P 3$, we have $e(f(A, C)) \leqslant$ $\min (e(f(A, B)), e(f(B, C)))$. Therefore, $N(A, C) \leqslant \min (N(A, B), N(B, C))$. 
Remark 2. In Proposition 11, let $\mu(x)=1-\frac{1}{2} x$, $E(x, y)=1-|x-y|^{n}$, then we have $f(A, B)(x)=$ $\frac{1+|A(x)-B(x)|^{n}}{2}$. Thus the similarity measure $e(f(A, B))$ is in accord with the one given in ${ }^{32}$.

Example 5. Consider the fuzzy equivalence $E(x, y)=\varphi^{-1}(1-|\varphi(x)-\varphi(y)|)$ defined in Proposition 2 , where $\varphi$ is an automorphism of the unit interval. Suppose $\mu(x)=1-\frac{1}{2} x$, then we have $f(A, B)(x)=$ $1-\frac{1}{2} \varphi^{-1}(1-|\varphi(A(x))-\varphi(B(x))|)$. Consider the following fuzzy entropy:

$$
e_{1}(A)=\frac{2}{n} \sum_{i=1}^{n} \min \left(A\left(x_{i}\right), 1-A\left(x_{i}\right)\right) .
$$

By Proposition 11, we obtain the following similarity measure:

$$
N_{6}(A, B)=\frac{1}{n} \sum_{i=1}^{n} \varphi^{-1}\left(1-\left|\varphi\left(A\left(x_{i}\right)\right)-\varphi\left(B\left(x_{i}\right)\right)\right|\right) .
$$

Note that $N_{6}$ is a similarity measure constructed in 7 .

Let $E$ be a fuzzy equivalence, $\lambda$ a strictly increasing function from $[0,1]$ to $\left[0, \frac{1}{2}\right]$ with boundary conditions $\lambda(0)=0, \lambda(1)=\frac{1}{2}$. For fuzzy sets $A$ and $B$, we define $g(A, B) \in F(X)$, for all $x \in X$, $g(A, B)(x)=\lambda(E(A(x), B(x)))$, then we have the following conclusion.

Proposition 12. Given a discrete universe $X=$ $\left\{x_{1}, x_{2}, \ldots, x_{n}\right\}$. Let e be a fuzzy entropy, $N$ a function defined for all $A, B \in F(X)$ by $N(A, B)=e(g(A, B))$, then $N$ is a similarity measure.

Proof. It can be proved in the same manner with Proposition 11.

Let $E$ be a fuzzy equivalence satisfying E5 and E6, $\mu$ and $\lambda$ the functions defined as above. For fuzzy set $A$, we define $p(A), q(A) \in F(X)$, for all $x \in X, p(A)(x)=f\left(A, A^{c}\right)(x)=\mu\left(E\left(A(x), A^{c}(x)\right)\right)$, $q(A)(x)=g\left(A, A^{c}\right)(x)=\lambda\left(E\left(A(x), A^{c}(x)\right)\right)$. By the definitions of $\mu$ and $\lambda$ we know that $q(A)(x) \leqslant$ $p(A)(x)$ for all $x \in X$, i.e., $q(A) \subseteq p(A)$. We have the following conclusion.
Proposition 13. Given a discrete universe $X=$ $\left\{x_{1}, x_{2}, \ldots, x_{n}\right\}$. Let $N$ be a similarity measure satisfying $N 5$ and N6, e a function defined for all $A \in F(X)$ by $e(A)=N(p(A), q(A))$, then $e$ is a fuzzy entropy.

\section{Proof.}

(EP1) (Necessity) If $N(p(A), q(A))=e(A)=0$, then by N5, $p(A) \cap q(A)=\emptyset$. Since $q(A) \subseteq p(A)$, we have $q(A)(x)=\lambda\left(E\left(A(x), A^{c}(x)\right)\right)=0$ for all $x \in X$. Since $\lambda$ is a strictly increasing function satisfying $\lambda(0)=0$, then $E\left(A(x), A^{c}(x)\right)=0$. As $E$ satisfies E6 we have $A(x)=1$ or $A(x)=0$. Therefore, $A$ is nonfuzzy.

(Sufficiency) If $A$ is nonfuzzy, then we have $A(x)=1$ or $A(x)=0$ for all $x \in X$. Thus $E\left(A(x), A^{c}(x)\right)=0$. This means that $q(A)(x)=$ $\lambda(0)=0, p(A)(x)=\mu(0)=1$ for all $x \in X$. Therefore, $q(A)=\emptyset, p(A)=X$. We have $e(A)=$ $N(p(A), q(A))=N(X, \emptyset)=0$.

(EP2) (Necessity) If $N(p(A), q(A))=e(A)=1$, then by N6, $p(A)=q(A)$. Therefore, $p(A)(x)=$ $\mu\left(E\left(A(x), A^{c}(x)\right)\right)=\lambda\left(E\left(A(x), A^{c}(x)\right)\right)=$ $q(A)(x)$ for all $x \in X$. By the properties of $\mu$ and $\lambda$, we have $E\left(A(x), A^{c}(x)\right)=1$. As $E$ satisfies E6 we have $A(x)=A^{c}(x)$, that is to say, $A(x)=\frac{1}{2}$.

(Sufficiency) If $A=\left[\frac{1}{2}\right]$, then $A(x)=\frac{1}{2}$ for all $x \in X$. Thus we conclude that $E\left(A(x), A^{c}(x)\right)=$ $E\left(\frac{1}{2}, \frac{1}{2}\right)=1$. This means that $q(A)(x)=\lambda(1)=$ $\frac{1}{2}, p(A)(x)=\mu(1)=\frac{1}{2}$ for all $x \in X$. Therefore, $p(A)=q(A), e(A)=N(p(A), q(A))=1$.

(EP3) For $x \in X$, if $A(x) \geqslant B(x) \geqslant \frac{1}{2}$, then $A^{c}(x) \leqslant$ $B^{c}(x) \leqslant \frac{1}{2}$, thus $A^{c}(x) \leqslant B^{c}(x) \leqslant \frac{1}{2} \leqslant B(x) \leqslant$ $A(x)$. By $E 4$ we have $E\left(A(x), A^{c}(x)\right) \leqslant$ $E\left(B(x), B^{c}(x)\right) . \quad B y$ the properties of $\mu$ and $\lambda, \quad \lambda\left(E\left(A(x), A^{c}(x)\right)\right) \leqslant \lambda\left(E\left(B(x), B^{c}(x)\right)\right) \leqslant$ $\mu\left(E\left(B(x), B^{c}(x)\right)\right) \leqslant \mu\left(E\left(A(x), A^{c}(x)\right)\right) . \quad$ This means that $q(A) \subseteq q(B) \subseteq p(B) \subseteq p(A)$. Thus $N(p(A), q(A)) \leqslant N(p(B), q(A)) \leqslant$ $N(p(B), q(B))$. That is to say, $e(A) \leqslant e(B)$. The case of $A(x) \leqslant B(x) \leqslant \frac{1}{2}$ can be proved similarly.

(EP4) Since $p(A)=p\left(A^{c}\right), q(A)=q\left(A^{c}\right)$, then $e(A)=$ $N(p(A), q(A))=N\left(p\left(A^{c}\right), q\left(A^{c}\right)\right)=e\left(A^{c}\right)$. 


\section{Remark 3.}

(1) In the conditions of Proposition 13, let $\mu(x)=1-\frac{1}{2} x, \lambda(x)=\frac{1}{2} x, E(x, y)=1-\mid x-$ $\left.y\right|^{n}$, then we have $p(A)(x)=\frac{1+\left|A(x)-A^{c}(x)\right|^{n}}{2}$, $q(A)(x)=\frac{1-\left|A(x)-A^{c}(x)\right|^{n}}{2}$. Thus the fuzzy entropy $N(p(A), q(A))$ is in accord with the one given by Zeng et al. ${ }^{32}$.

(2) In conditions of (1), if $n=1$, then we have $p(A)(x)=\frac{1+\left|A(x)-A^{c}(x)\right|}{2}=\max \left(A(x), A^{c}(x)\right)$, $q(A)(x)=\frac{1-\left|A(x)-A^{c}(x)\right|}{2}=\min \left(A(x), A^{c}(x)\right)$. This means that $p(A)=A \cup A^{c}, q(A)=A \cap A^{c}$. Thus the fuzzy entropy constructed by similarity measure $N$ can be expressed as $N(A \cup$ $A^{c}, A \cap A^{c}$ ). This is the solution given by Fan in ${ }^{13}$.

Example 6. Suppose $\mu(x)=1-\frac{1}{2} x, \lambda(x)=\frac{1}{2} x$, $E(x, y)=\varphi^{-1}(1-|\varphi(x)-\varphi(y)|)$, where $\varphi$ is an automorphism of the unit interval. Since $E$ is a fuzzy equivalence satisfying $E 5$ and $E 6$, we have

$$
\begin{aligned}
q(A)(x) & =\lambda\left(E\left(A(x), A^{c}(x)\right)\right) \\
& =\frac{1}{2} \varphi^{-1}(1-|\varphi(A(x))-\varphi(1-A(x))|), \\
p(A)(x) & =\mu\left(E\left(A(x), A^{c}(x)\right)\right) \\
& =1-\frac{1}{2} \varphi^{-1}(1-|\varphi(A(x))-\varphi(1-A(x))|) .
\end{aligned}
$$

Consider the following similarity measure:

$$
N_{2}(A, B)=\frac{\sum_{i=1}^{n} \min \left(A\left(x_{i}\right), B\left(x_{i}\right)\right)}{\sum_{i=1}^{n} \max \left(A\left(x_{i}\right), B\left(x_{i}\right)\right)} .
$$

It is shown that $N_{2}$ satisfies properties N4, N5, N6, by Proposition 13, we obtain the following fuzzy entropy:

$e_{2}(A)=\frac{\sum_{i=1}^{n} \varphi^{-1}\left(1-\left|\varphi\left(A\left(x_{i}\right)\right)-\varphi\left(1-A\left(x_{i}\right)\right)\right|\right)}{\sum_{i=1}^{n}\left(2-\varphi^{-1}\left(1-\left|\varphi\left(A\left(x_{i}\right)\right)-\varphi\left(1-A\left(x_{i}\right)\right)\right|\right)\right)}$.

Proposition 14. Given a discrete universe $X=$ $\left\{x_{1}, x_{2}, \ldots, x_{n}\right\}$. Let $N$ be a similarity measure satisfying $N 5$ and N6, e a function defined for all $A \in F(X)$ by $e(A)=N\left(A, A^{c}\right)$, then e is a fuzzy entropy.
Proof. It follows from Proposition 13 that this proposition holds.

\section{Relation between subsethood measure and fuzzy entropy}

At first blush, subsethood measure and fuzzy entropy do not seem related. To relate subsethood measure with fuzzy entropy, Kosko ${ }^{18}$ proposed the following expression: given a subsethood measure $c$, the fuzzy entropy $e$ generated by $c$ is defined as $e(A)=c\left(A \cup A^{c}, A \cap A^{c}\right)$ for all $A \in F(X)$. For showing the conditions of $c$ for which $e$ can be a fuzzy entropy, several axiomatizations were given in the literature. In the previous part of this paper, we have referred to three concepts of subsethood measure, that is, $V Y$-subsethood measure, *-subsethood measure, and $D I$-subsethood measure. It is shown that all $D I$-subsethood measure is $V Y$-subsethood measure and therefore, it is also $*$-subsethood measure. We know that the three conditions of $*$-subsethood measure are enough to demand the conditions for the expression: $e(A)=c\left(A \cup A^{c}, A \cap A^{c}\right)$ to fulfill the conditions demanded from fuzzy entropy. Therefore, we use $*$-subsethood measure to construct fuzzy entropy here.

Proposition 15. Given a discrete universe $X=$ $\left\{x_{1}, x_{2}, \ldots, x_{n}\right\}$. Let $c$ be a $*$-subsethood measure, $p(A)$ defined as above. Suppose $(p(A))^{c}$ is the complement of the fuzzy set $p(A)$. If $e$ is a function defined for all $A \in F(X)$ by $e(A)=c\left(p(A),(p(A))^{c}\right)$, then $e$ is a fuzzy entropy.

\section{Proof.}

(EP1) (Necessity) Since $p(A)(x) \geqslant \frac{1}{2}$, then we have $\left[\frac{1}{2}\right] \subseteq p(A)$. If $c\left(p(A),(p(A))^{c}\right)=0$, then by $C 2$ of $*$-subsethood measure, we have $p(A)=X$. This means that $p(A)(x)=\mu\left(E\left(A(x), A^{c}(x)\right)\right)=$ 1 for all $x \in X$. Thus according to the properties of $\mu$ we have $E\left(A(x), A^{c}(x)\right)=0$. As $E$ satisfies E6 we have $A(x)=1$ or $A(x)=0$. Therefore, $A$ is nonfuzzy.

(Sufficiency) $A$ is nonfuzzy implies $A(x)=1$ or $A(x)=0$ for all $x \in X$. This means that $E\left(A(x), A^{c}(x)\right)=0 . \quad$ Thus $p(A)(x)=\mu(0)=$ 
$1, p\left(A^{c}\right)(x)=0$, that is to say, $p(A)=X$. Therefore, we have $e(A)=c\left(p(A),(p(A))^{c}\right)=$ $c(X, \emptyset)=0$.

(EP2) (Necessity) If $c\left(p(A),(p(A))^{c}\right)=e(A)=1$, then by $C 1$ of $*$-subsethood measure, $p(A) \subseteq$ $(p(A))^{c}$. As $(p(A))^{c} \subseteq p(A)$ we have $(p(A))^{c}=$ $p(A)$. Thus $\mu\left(E\left(A(x), A^{c}(x)\right)\right)=\frac{1}{2}$ for all $x \in X$. According to the properties of $\mu$, we have $E\left(A(x), A^{c}(x)\right)=1$. As $E$ satisfies $E 5$ we have $A(x)=A^{c}(x)$, that is to say, $A(x)=\frac{1}{2}$.

(Sufficiency) If $A=\left[\frac{1}{2}\right]$, then $A(x)=\frac{1}{2}$ for all $x \in X$. Thus we conclude that $E\left(A(x), A^{c}(x)\right)=$ 1. This means that $p(A)(x)=(p(A))^{c}(x)=$ $\frac{1}{2}$, i.e., $p(A)=(p(A))^{c}$. Therefore, $e(A)=$ $c\left(p(A),(p(A))^{c}\right)=1$.

(EP3) For $x \in X$, if $A(x) \leqslant B(x) \leqslant \frac{1}{2}$, then $A^{c}(x) \geqslant$ $B^{c}(x) \geqslant \frac{1}{2}$, thus $A(x) \leqslant B(x) \leqslant \frac{1}{2} \leqslant B^{c}(x) \leqslant$ $A^{c}(x)$. By E4, we have $E\left(A(x), A^{c}(x)\right) \leqslant$ $E\left(B(x), B^{c}(x)\right)$. By the properties of $\mu$, we have $1-\mu\left(E\left(A(x), A^{c}(x)\right)\right) \leqslant 1-\mu\left(E\left(B(x), B^{c}(x)\right)\right) \leqslant$ $\mu\left(E\left(B(x), B^{c}(x)\right)\right) \leqslant \mu\left(E\left(A(x), A^{c}(x)\right)\right) . \quad$ This means that $(p(A))^{c} \subseteq(p(B))^{c} \subseteq p(B) \subseteq$ $p(A)$. By $C 3$ of $*$-subsethood measure, we have $c\left(p(A),(p(A))^{c}\right) \leqslant c\left(p(B),(p(A))^{c}\right) \leqslant$ $c\left(p(B),(p(B))^{c}\right)$. Therefore, $e(A) \leqslant e(B)$. The case of $A(x) \geqslant B(x) \geqslant \frac{1}{2}$ can be proved similarly.

(EP4) By the definition of $p(A)$, we have $p(A)=$ $p\left(A^{c}\right)$. Therefore, $e(A)=c\left(p(A),(p(A))^{c}\right)=$ $c\left(p\left(A^{c}\right),\left(p\left(A^{c}\right)\right)^{c}\right)=e\left(A^{c}\right)$.

Remark 4. In the conditions of Proposition 15 , let $\mu(x)=1-\frac{1}{2} x, E(x, y)=1-|x-y|$, then we have $p(A)(x)=\frac{1+\left|A(x)-A^{c}(x)\right|}{2}=\max \left(A(x), A^{c}(x)\right)$, $(p(A))^{c}(x)=\frac{1-\left|A(x)-A^{c}(x)\right|^{2}}{2}=\min \left(A(x), A^{c}(x)\right)$. This means that $p(A)=A \cup A^{c},(p(A))^{c}=A \cap A^{c}$. Thus the fuzzy entropy derived from subsethood measure $c$ can be expressed as $c\left(A \cup A^{c}, A \cap A^{c}\right)$. In this sense, Kosko's solution ${ }^{18}$ about the relation between fuzzy entropy and subsethood measure can be brought into line with our solution.
Example 7. Suppose $\mu(x)=1-\frac{1}{2} x, \quad E(x, y)=$ $\varphi^{-1}\left(\frac{\min (\varphi(x), \varphi(y))}{\max (\varphi(x), \varphi(y))}\right)$, where $\varphi$ is an automorphism of the unit interval. According to Proposition 3, we know that $E$ is a fuzzy equivalence satisfying $E 5$ and $E 6$, then we have

$$
\begin{aligned}
p(A)(x) & =\mu\left(E\left(A(x), A^{c}(x)\right)\right) \\
& =1-\frac{1}{2} \varphi^{-1}\left(\frac{\min (\varphi(A(x)), \varphi(1-A(x)))}{\max (\varphi(A(x)), \varphi(1-A(x)))}\right), \\
(p(A))^{c}(x) & =1-\mu\left(E\left(A(x), A^{c}(x)\right)\right) \\
& =\frac{1}{2} \varphi^{-1}\left(\frac{\min (\varphi(A(x)), \varphi(1-A(x)))}{\max (\varphi(A(x)), \varphi(1-A(x)))}\right) .
\end{aligned}
$$

Consider the following subsethood measure:

$$
c_{6}(A, B)=\frac{\sum_{i=1}^{n} B\left(x_{i}\right)}{\sum_{i=1}^{n} \max \left(A\left(x_{i}\right), B\left(x_{i}\right)\right)} .
$$

It is shown that $c_{6}$ is a $*$-subsethood measure. By Proposition 15, we obtain the following fuzzy entropy:

$$
e_{3}(A)=\frac{\sum_{i=1}^{n} \varphi^{-1}\left(\frac{\min \left(\varphi\left(A\left(x_{i}\right)\right), \varphi\left(1-A\left(x_{i}\right)\right)\right)}{\max \left(\varphi\left(A\left(x_{i}\right)\right), \varphi\left(1-A\left(x_{i}\right)\right)\right)}\right)}{\sum_{i=1}^{n}\left(2-\varphi^{-1}\left(\frac{\min \left(\varphi\left(A\left(x_{i}\right)\right), \varphi\left(1-A\left(x_{i}\right)\right)\right)}{\max \left(\varphi\left(A\left(x_{i}\right)\right), \varphi\left(1-A\left(x_{i}\right)\right)\right)}\right)\right)} .
$$

Let $E$ be a fuzzy equivalence satisfying E5 and E6, $\mu$ and $\lambda$ the functions defined as above. For fuzzy sets $A$ and $B$, we define $k(A, B), l(A, B) \in F(X)$, for all $x \in X$, $k(A, B)(x)=\mu(E(A(x), \min (A(x), B(x)))), l(A, B)(x)=$ $\lambda(E(A(x), \min (A(x), B(x))))$. Then we have the following conclusion.

Proposition 16. Given a discrete universe $X=$ $\left\{x_{1}, x_{2}, \ldots, x_{n}\right\}$. Let e be a fuzzy entropy, $c$ a function defined for all $A, B \in F(X)$ by $c(A, B)=e(k(A, B))$, then $c$ is a DI-subsethood measure.

\section{Proof.}

(C1) (Necessity) If $c(A, B)=e(k(A, B))=1$, by $E P 2$, we have $k(A, B)=\left[\frac{1}{2}\right]$. This means that $\mu(E(A(x), \min (A(x), B(x))))=\frac{1}{2}$ for all $x \in X$. Since $\mu$ is a strictly decreasing function and $\mu(1)=\frac{1}{2}$, then we have $E(A(x), \min (A(x), B(x)))=1$ for all $x \in X$. As $E$ satisfies $E 5$ we have $A(x)=\min (A(x), B(x))$, thus $A(x) \leqslant B(x)$ for all $x \in X$. 
(Sufficiency) If $A \subseteq B$, then $k(A, B)(x)=$ $\mu(E(A(x), A(x)))=\mu(1)=\frac{1}{2}$, that is to say, $k(A, B)=\left[\frac{1}{2}\right]$. Therefore, $c(A, B)=e\left(\left[\frac{1}{2}\right]\right)=1$.

(C2) (Necessity) If $e\left(k\left(A, A^{c}\right)\right)=c\left(A, A^{c}\right)=0$, then by $E P 1, \quad k\left(A, A^{c}\right)$ is nonfuzzy. Therefore, $k\left(A, A^{c}\right)(x)=1$ or 0 . By the definition of $k(A, B)$, we know $k(A, B)(x) \geqslant$ $\frac{1}{2}$. Thus $k\left(A, A^{c}\right)(x)=1$ for all $x \in$ $X$. This means that $\mu(E(A(x), \min (A(x), 1-$ $A(x))))=1$. By the properties of $\mu$, we have $E(A(x), \min (A(x), 1-A(x)))=0$. If $A(x) \leqslant \frac{1}{2}$, then $E(A(x), \min (A(x), 1-A(x)))=$ $E(A(x), A(x))=1 \neq 0$. Thus we conclude that $A(x) \geqslant \frac{1}{2}$ for all $x \in X$. Thus $E(A(x), 1-$ $A(x))=0$. As $E$ satisfies E6 we have $A(x)=1$ for all $x \in X$.

(Sufficiency) If $A=X$, then $k\left(A, A^{c}\right)(x)=$ $\mu(E(1,0))=\mu(0)=1$. Thus $k\left(A, A^{c}\right)=X$. Therefore, $c\left(A, A^{c}\right)=e\left(k\left(A, A^{c}\right)\right)=e(X)=0$.

(C3) Since $A \subseteq B$ implies $A(x) \leqslant B(x)$ for all $x \in X$, then for any fuzzy set $C$, three cases will be considered depending on the position of $C(x)$.

(1) If $A(x) \leqslant B(x) \leqslant C(x)$, then $k(B, C)(x)=$ $\mu(E(B(x), B(x)))=\mu(1)=\frac{1}{2}, k(A, C)(x)=$ $\mu(E(A(x), A(x)))=\mu(1)=\frac{1}{2}$ for $x \in X$. Thus, $k(B, C)(x)=k(A, C)(x)$.

(2) If $C(x) \leqslant A(x) \leqslant B(x)$, then $k(B, C)(x)=$ $\mu(E(B(x), C(x))), k(A, C)(x)=\mu(E(A(x), C(x)))$ for $x \in X$. According to E4, we conclude that $E(B(x), C(x)) \leqslant E(A(x), C(x))$. As $\mu$ is strictly decreasing we have $\mu(E(B(x), C(x))) \geqslant \mu(E(A(x), C(x)))$. Thus, $k(B, C)(x) \geqslant k(A, C)(x)$.

(3) If $A(x) \leqslant C(x) \leqslant B(x)$, then $k(B, C)(x)=$ $\mu(E(B(x), C(x))), k(A, C)(x)=\mu(E(A(x), A(x)))$ for $x \in X$. Since $E(B(x), C(x)) \leqslant$ $E(A(x), A(x))=1$ and $\mu$ is strictly decreasing, then we have $\mu(E(B(x), C(x))) \geqslant$ $\mu(E(A(x), A(x)))$. Therefore, $k(B, C)(x) \geqslant$ $k(A, C)(x)$.

Hence we can conclude that $k(B, C)(x) \geqslant$ $k(A, C)(x) \geqslant \frac{1}{2}$ for all $x \in X$. Then by EP3 we have $e(k(B, C)) \leqslant e(k(A, C))$, i.e., $c(B, C) \leqslant$
$c(A, C)$. The case of $c(C, A) \leqslant c(C, B)$ whenever $A \subseteq B$ can be proved similarly.

Example 8. In the conditions of Proposition 16, let $\mu(x)=1-\frac{1}{2} x, E(x, y)=\varphi^{-1}\left(\frac{\min (\varphi(x), \varphi(y))}{\max (\varphi(x), \varphi(y))}\right)$, where $\varphi$ is an automorphism of the unit interval, then we have $k(A, B)(x)=1-\frac{1}{2} \varphi^{-1}\left(\frac{\varphi(\min (A(x), B(x)))}{\varphi(A(x))}\right)$. Consider the following fuzzy entropy:

$$
e_{1}(A)=\frac{2}{n} \sum_{i=1}^{n} \min \left(A\left(x_{i}\right), 1-A\left(x_{i}\right)\right) .
$$

By Proposition 16, we obtain the following $D I$ subsethood measure:

$$
c_{7}(A, B)=\frac{1}{n} \sum_{i=1}^{n} \varphi^{-1}\left(\frac{\varphi\left(\min \left(A\left(x_{i}\right), B\left(x_{i}\right)\right)\right)}{\varphi\left(A\left(x_{i}\right)\right)}\right) .
$$

Proposition 17. Given a discrete universe $X=$ $\left\{x_{1}, x_{2}, \ldots, x_{n}\right\}$. Let e be a fuzzy entropy, $c$ a function defined for all $A, B \in F(X)$ by $c(A, B)=e(l(A, B))$, then $c$ is a DI-subsethood measure.

Proof. It can be proved in the same manner with Proposition 16.

Corollary 18. The subsethood measures constructed by fuzzy entropy in the above-mentioned propositions are also $V Y$-subsethood measures and therefore, they are also *-subsethood measures.

\section{Conclusions}

On the basis of the definition of similarity measure proposed by Wang ${ }^{26}$, the definition of $V Y$ subsethood measure ${ }^{30}, *$-subsethood measure ${ }^{11}$ and $D I$-subsethood measure ${ }^{4}$, and the definition of fuzzy entropy introduced by De Luca and Termini ${ }^{9}$, we have investigated the relations among similarity measure, subsethood measure and fuzzy entropy. We also have presented several propositions that similarity measure, subsethood measure and fuzzy entropy can be transformed by each other based on their axiomatic definitions. It is shown that the results obtained in the literature can be brought into line with the present work. 


\section{Acknowledgments}

This work is partially supported by the National Natural Science Foundation of China (Grant Nos. 61175044, 61175055, 61100046), the 2013 Doctoral Innovation Funds of Southwest Jiaotong University, and the Fundamental Research Funds for the Central University.

\section{References}

1. W. Bandler, L. Kohout, Fuzzy power sets and fuzzy implication operators, Fuzzy Sets and Systems 4 (1980) 13-30.

2. P. Burillo, H. Bustince, Entropy on intuitionistic fuzzy sets and on interval-valued fuzzy sets, Fuzzy Sets and Systems 78 (1996) 305-316.

3. H. Bustince, P. Burillo, F. Soria, Automorphisms, negations and implication operators, Fuzzy Sets and Systems 134 (2003) 209-229.

4. H. Bustince, V. Mohedano, E. Barrenechea, M. Pagola, Definition and construction of fuzzy DIsubsethood measures, Information Sciences 176 (2006) 3190-3231.

5. H. Bustince, E. Barrenechea, M. Pagola, Restricted equivalence functions, Fuzzy Sets and Systems 157 (2006) 2333-2346.

6. H. Bustince, E. Barrenechea, M. Pagola, Image thresholding using restricted equivalence functions and maximizing the measures of similarity, Fuzzy Sets and Systems 158 (2007) 496-516.

7. H. Bustince, E. Barrenechea, M. Pagola, Relationship between restricted dissimilarity functions, restricted equivalence functions and normal $E_{N}$-functions: Image thresholding invariant, Patter Recognition Letters 29 (2008) 525-536.

8. S.M. Chen, M.S. Yeh, P.Y. Hsiao, A comparison of similarity measures of fuzzy values, Fuzzy Sets and Systems 72 (1995) 79-89.

9. A. De Luca, S. Termini, A definition of a nonprobabilistic entropy in the setting of fuzzy sets, Information and Control 20 (1972) 301-312.

10. D. Dubois, H. Prade, Fuzzy Sets and Systems: Theory and Applications. Academic Press, New York (1980).

11. J.L. Fan, W.X. Xie, Some notes on similarity measure and proximate measure, Fuzzy Sets and Systems 101 (1999) 403-412.

12. J.L. Fan, W.X. Xie, J. Pei, Subsethood measures: new definitions, Fuzzy Sets and Systems 106 (1999) 201209.

13. J.L. Fan, Subsethood measures, Northwestern Press,
Hsian, 1999. (in Chinese)

14. J.C. Fodor, M. Roubens, Fuzzy Preference Modelling and Multicriteria Decision Support, Kluwer Academic Publishers, Dordrecht (1994).

15. A. Kaufmann, Introduction to the Theory of Fuzzy Subsets. Academic Press, New York (1975).

16. L. Kitainik, Fuzzy inclusions and fuzzy dichotomous decision procedures, J. Kacprzyk, S. Orlovski, Editors , Optimization Models Using Fuzzy Sets and Possibility Theory, Reidel, Dordrecht (1987) 154-170.

17. E.P. Klement, R. Mesiar, E. Pap, Triangular Norms, Kluwer Academic Publishers, Dordrecht (2000).

18. B. Kosko, Fuzzy entropy and conditioning. Information Sciences 40 (1986) 165-174.

19. B. Kosko, Fuzziness vs. probability, International Journal of General Systems 17 (1990) 211-240.

20. B. Kosko, Neural Networks and Fuzzy Systems: A Dynamical Systems Approach to Machine Intelligence. Prentice Hall, Englewood Cliffs (1992).

21. X.C. Liu, Entropy, distance measure and similarity measure of fuzzy sets and their relations, Fuzzy Sets and Systems 52 (1992) 305-318.

22. S.G. Loo, Measures of fuzziness, Cybernetica 20 (1977) 201-210.

23. C.P. Pappis, N.I. Karacapilidis, A comparative assessment of measures of similarity of fuzzy values, Fuzzy Sets and Systems 56 (1993) 171-174.

24. D. Sinha, E.R. Dougherty, Fuzzification of set inclusion: Theory and applications, Fuzzy Sets and Systems 55 (1993) 15-42.

25. E. Trillas, T. Riera, Entropies in finite fuzzy sets, Information Sciences 15 (1978) 159-168.

26. P.Z. Wang, Fuzzy Sets and Its Applications, Shanghai Science and Technology Press, Shanghai, 1983. (in Chinese)

27. X.Z. Wang, B.D. Baets, E.E. Kerre, A comparative study of similarity measures, Fuzzy Sets and Systems 73 (1995) 259-268.

28. R. Willmott, On the transitivity of containment and equivalence in fuzzy power set theory, Journal of Mathematical Analysis and Applications 120 (1986) 384-396.

29. R.R. Yager, On the measure of fuzziness and negation. Part I: Membership in the unit interval. International Journal of General Systems 5 (1979) 189-200.

30. V.R. Young, Fuzzy subsethood, Fuzzy Sets and Systems 77 (1996) 371-384.

31. L.A. Zadeh, Fuzzy sets, Information Control 8 (1965) 338-353.

32. W.Y. Zeng, H.X. Li, Inclusion measure, similarity measure and the fuzziness of fuzzy sets and their relations, International Journal of Intelligent Systems 21 (2006) 639-653. 